EPJ Web of Conferences 59, 03007 (2013)

DOI: $10.1051 /$ epjconf/20135903007

(C) Owned by the authors, published by EDP Sciences, 2013

\title{
Optimum design of imploded core plasma for effective fast ignition at GXII
}

\author{
H. Nagatomo ${ }^{1, a}$, T. Johzaki ${ }^{2}$, A. Sunahara ${ }^{2}$, H. Sakagami ${ }^{3}$, T. Yanagawa ${ }^{4}$ \\ and K. Mima ${ }^{5}$ \\ ${ }^{1}$ Institute of Laser Engineering, Osaka University, 2-6 Yamada-oka, Suita, Osaka, Japan \\ ${ }^{2}$ Institute for Laser Technology, 2-6 Yamada-oka, Suita, Osaka, Japan \\ ${ }^{3}$ National Institute for Fusion Science, Toki 509-5202, Japan \\ ${ }^{4}$ Department of Physics, Nagoya University, Nagoya 464-8602, Japan \\ ${ }^{5}$ Graduate School for the Creation of New Photonics Industries Hamamatsu, Hamamatsu, \\ Japan
}

\begin{abstract}
In the implosion phase of the fast ignition scheme, most critical issues are breakup of the cone tip and the formation of high $\rho$-R core plasma to improve its heating efficiency. For the integrated fast ignition experiment at ILE Osaka University, robust and reliable implosion must be redesign. In this paper, feasible target design under the constraint condition of existing GXII and LFEX facilities is studied using two-dimensional radiation hydrodynamic simulations, and an optimum target design based on low velocity implosion is proposed. The advantages of low velocity implosion are low adiabat, robust against RayleighTaylor instability, which are verified. Also longer life time of compressed core plasma which is preferable for fast ignition is confirmed in this study.
\end{abstract}

\section{INTRODUCTION}

The Fast ignition (FI) is one of the most attractive schemes in laser fusion. Mostly, a shell target for FI is fitted with reentrant gold cone target to preserve a path for heating laser. There were many integrated experiment using GXII laser for implosion and LFEX laser for heating in FIREX (Fast ignition realization experiment: phase-1) at Institute of Laser Engineering (ILE) Osaka University. The goal of FIREX-I project is achieving $5 \mathrm{keV}$ of ion temperature at compressed core. In these experiments, all the implosions were conventional scheme which was similar design as high density compression experiment for central ignition [1]. Target shell size was $250 \mu \mathrm{m}$ in radius, and $7 \mu \mathrm{m}$ thickness. Implosion velocity was reached to $3 \times 10^{7} \mathrm{~cm} / \mathrm{s}$ in order to achieve high temperature in the hot spot, which was appeared at the center of the implosion. For the high efficient fast ignition such conservative design must be revised. Optimum implosion design of cone guided shell target for FIREX-I is studied using two-dimensional radiation hydrodynamic simulation [2].

There are some theoretical design concepts for fast ignition [3, 4]. However there are technical limitations in these laser facilities, such as laser energy, laser uniformity, and pulse duration which are important parameter for optimization. In this study, 1-D implosion is optimized based on low velocity implosion [3] taking the constrain condition of FIREX-I laser facility into consideration. Then 2-D simulations are executed to verify the two dimensional dynamics of cone guided implosion, and understand characteristic of it. Furthermore hydrodynamic instability is seeded to verify the robustness

\footnotetext{
ae-mail: naga@ile.osaka-u.ac.jp
}

This is an Open Access article distributed under the terms of the Creative Commons Attribution License 2.0, which permits unrestricted use, distribution, and reproduction in any medium, provided the original work is properly cited. 
low velocity implosion target

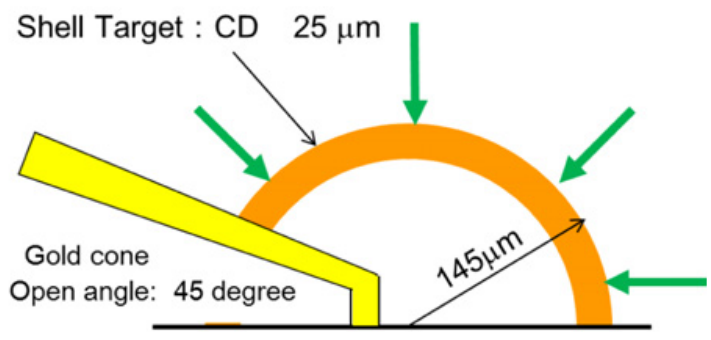

Figure 1. Configuration of cone-guided shell target.

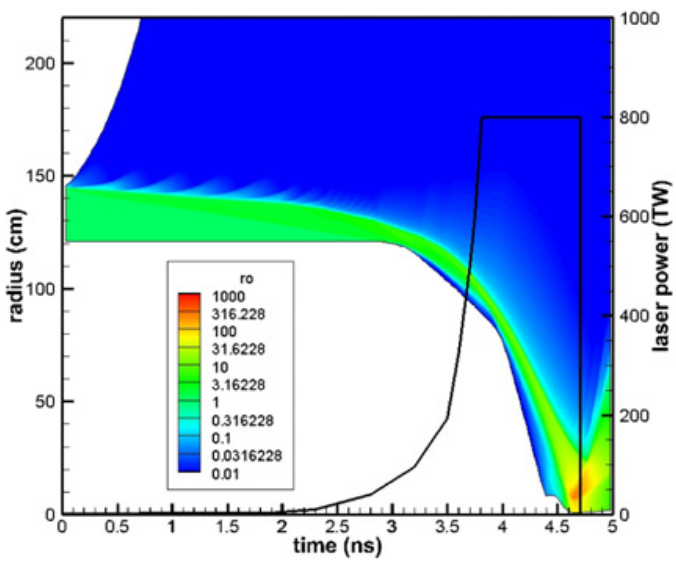

Figure 2. Optimized 1-D trajectory. Mass density contours $\left(\mathrm{g} / \mathrm{cm}^{3}\right)$ and laser power (right-axis).

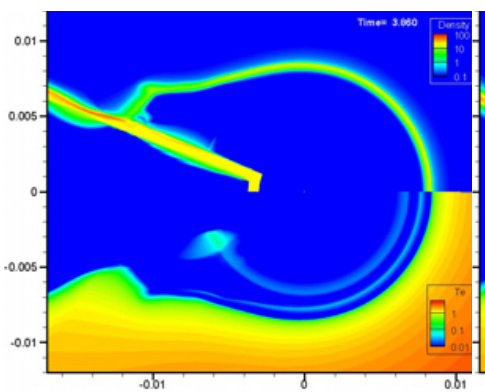

(a) $t_{\max }-500 \mathrm{ps}$

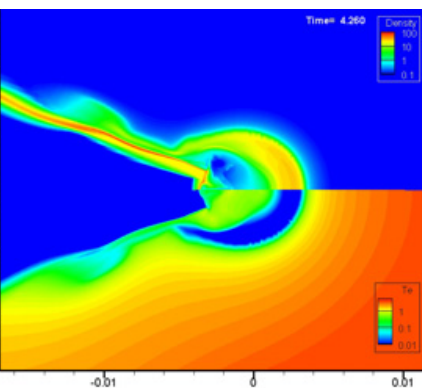

(b) $t_{\max }-100 \mathrm{ps}$

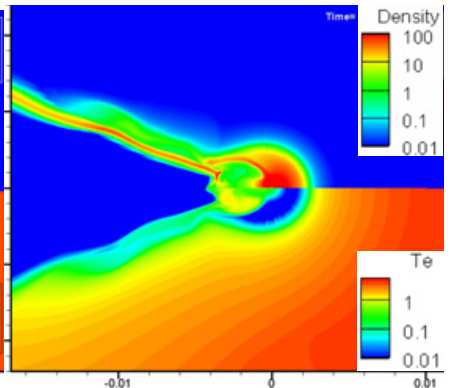

(c) $t_{\max }$

Figure 3. Mass density in $\mathrm{g} / \mathrm{cm}^{3}$ (top) and electron temperature in $\mathrm{keV}$ (bottom) contours at $500 \mathrm{ps,} 100 \mathrm{ps}$, and $0 \mathrm{ps}$ before the maximum compression time in the case of low velocity implosion with uniform laser irradiation.

as a preliminary check. Material and shape of tip should be carefully design for the improvement of heating efficiency of fast ignition as well. However, it can be designed separately and it is not discussed in this paper.

\section{SIMULATIONS}

The 2-D radiation hydrodynamic code, PINOCO [5] is used for these simulations. Simulation conditions are based on current direct drive implosion laser GXII performance. The total energy and the wavelength of the laser are assumed as $3.3 \mathrm{~kJ}$ and $0.53 \mu \mathrm{m}$ respectively. The tailored laser pulse is applied and its duration is limited to $4.5 \mathrm{~ns}$ due to the GXII limitation. In the conventional implosion, initial radius and thickness of the typical CD shell target are $250 \mu \mathrm{m}$ and $7 \mu \mathrm{m}$ respectively. Here, they are set to $145 \mu \mathrm{m}$ and $25 \mu \mathrm{m}$ (Fig. 1) respectively, according to the optimized 1-D design using 1-D implosion code, ILESTA-1D (Fig. 1), where the implosion velocity reaches $1.6 \times 10^{7} \mathrm{~cm} / \mathrm{s}$ which is half of conventional implosion. For the 2-D simulation, open angle of the cone is set to 45 degree. Distance between cone tip and the center of the shell is $30 \mu \mathrm{m}$.

The mass density and electron temperature contours of slow velocity implosion with uniform laser irradiation case are shown in Fig. 3. Averaged density, radius, and averaged ion temperature of core are 


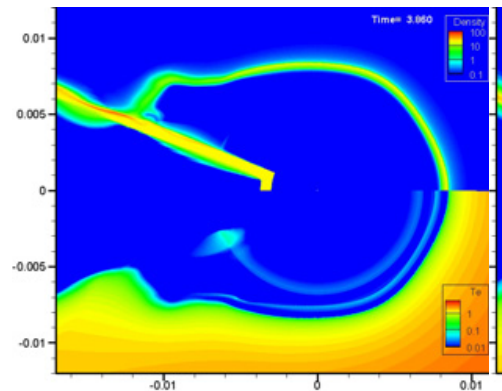

(a) $t_{\max }-500 \mathrm{ps}$

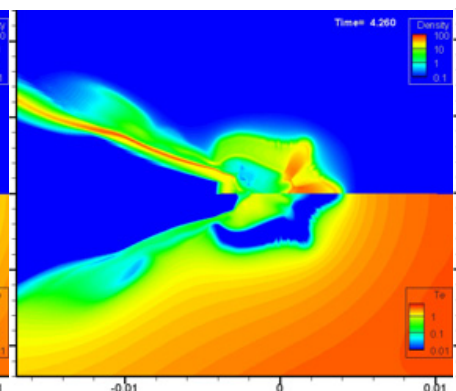

(b) $t_{\max }-100 \mathrm{ps}$

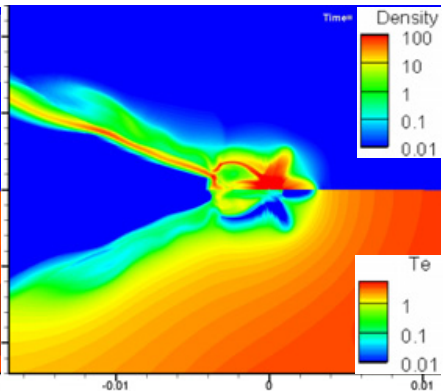

(c) $t_{\max }$

Figure 4. Mass density in $\mathrm{g} / \mathrm{cm}^{3}$ (top) and electron temperature in $\mathrm{keV}$ (bottom) contours at $500 \mathrm{ps}, 100 \mathrm{ps}$, and 0 ps before the maximum compression time in the case of low velocity implosion with non-uniform laser irradiation.

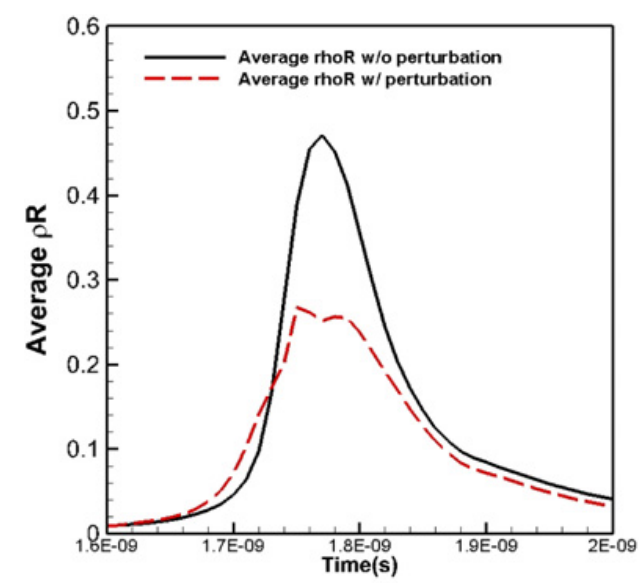

(a)

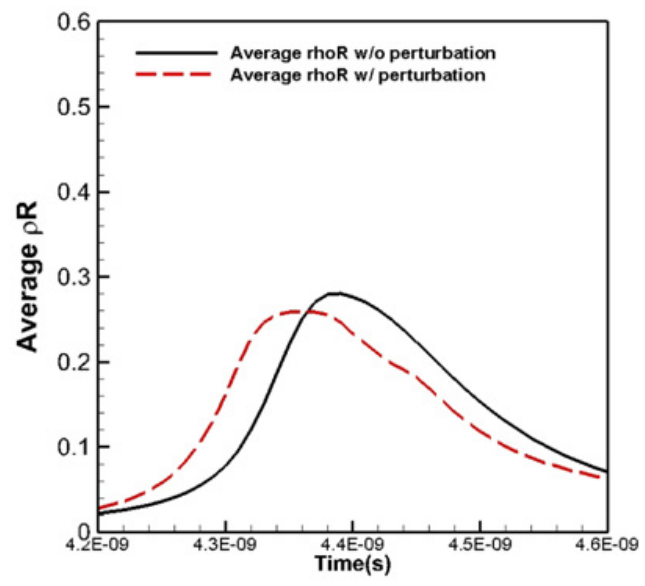

(b)

Figure 5. (a) Temporal averaged $\rho-\mathrm{R}\left(\mathrm{g} / \mathrm{cm}^{2}\right)$ of core plasma in conventional cone-guided implosion case, with laser non-uniformity (dash line) and without it (solid line). (b) Temporal averaged $\rho$ - $\mathrm{R}$ of core plasma in slow velocity implosion case, with laser non-uniformity (dash line) and without it (solid line).

$126 \mathrm{~g} / \mathrm{cm}^{3}, 24 \mu \mathrm{m}$ and $30 \mathrm{eV}$ respectively. Though high density core is form successfully, there are some points to be noticed. The CD shell near the gold cone is not accelerated effectively, and stranded. And gold cone is pressurized by ablated plasma and close to be broken near the initial contact position of gold cone and CD shell. This damage is not fatal, but it should be paid attention.

The robustness of the implosion is required against laser non-uniform irradiation, target surface roughness, and pulse shaping. In this study non-uniformity of laser irradiation are examined, where nonuniformity of mode 6 and amplitude of 5\% are assumed. They are typical numbers in GXII operation. This simulation result is shown in Fig 4. The in-flight aspect ratio is small, and the acceleration is not so high to break the shell before the maximum compression due to the Rayleigh-Taylor instability. On the contrary, the shell is almost broken up if conventional implosion scheme is applied. This thick shell target is a significant advantage of this scheme.

Figure 5 shows the temporal averaged $\rho$-R of compressed core plasma in cases of (a) conventional implosion and (b) slow implosion respectively. The solid lines and dashed lines indicate the implosion with uniform laser irradiation and with non-uniform laser irradiation respectively. In this ideal case $\rho$-R is $0.48 \mathrm{~g} / \mathrm{cm}^{2}$ with uniform laser irradiation whereas $0.26 \mathrm{~g} / \mathrm{cm}^{2}$ with non-uniform laser irradiation. On 
the contrary, in low velocity implosion cases, they are about $0.30 \mathrm{~g} / \mathrm{cm}^{2}$ and $0.26 \mathrm{~g} / \mathrm{cm}^{2}$. In realistic conditions, the fatal higher mode of laser non-uniformity and target surface roughness which are not taken account in these simulations must be seeded furthermore. This target design will be effective against such perturbations also.

The duration of high $\rho$-R period $\left(\rho-\mathrm{R}>0.2 \mathrm{~g} / \mathrm{cm}^{2}\right)$ is $80 \mathrm{ps}$ in conventional implosion case and $140 \mathrm{ps}$ in slow implosion velocity case respectively. This is another advantage of low velocity implosion, because synchronizing laser pulse error of GXII and LFEX is a few tens pico second and longer heating window is preferable in view point of heating process.

The breakup time of the tip is $4.38 \mathrm{~ns}$ which is almost the same as the maximum compression time in case of Au tip (thickness: $6 \mu \mathrm{m}$ ). In this study, tip material, shape, position, and thickness are not optimized yet. It can be delayed if heavier material or thicker tip is applied. This optimization problem has a close relation to high energy electron transport matter. One solution is application of our recent simulation studies of pointed-cone-tip concept using Diamond-like-Carbon [5]. It shows that breakup of the tip can be delayed after maximum compression, and to create the optimum shape for guiding high energy electron transport toward the imploded core.

\section{CONCLUSION}

The slow velocity implosion for fast ignition is studied, and an optimized design for FIREX-I experiment is proposed using 2-D radiation hydrodynamic simulations. The advantage of the scheme is not only low adiabat implosion but also robust against hydrodynamic instability caused by the non-uniform laser irradiation and target surface roughness. Furthermore, we have found that relatively longer confinement time may extend the heating window time in this scheme.

The authors are grateful for the support of the computer room of ILE and the Cyber-Media Center at Osaka University.

\section{References}

[1] H. Azechi et al., Laser Part. Beams, 9, No. 2, 193 (1991)

[2] H. Nagatomo et al., Phys. Plasmas 14, 056303 (2007)

[3] R. Betti and C. Zhou, Phys. Plasmas 12, 110702 (2005)

[4] D.S. Clark and M. Tabak, Nucl. Fusion 47, 1147-1156 (2007)

[5] A. Sunahara et al., Laser Part. Beams, to be published. 